\title{
ESSENTIALLY COMPACT FOLIATIONS THAT ARE NOT COMPACT
}

\author{
TRACY L. PAYNE
}

(Received October 30, 2002)

\begin{abstract}
We study smooth foliations of compact manifolds where the set of noncompact leaves has zero Lebesgue measure (and is nonempty). We review a simple example due to Reeb and provide two new examples that show more complicated behavior that may occur.
\end{abstract}

1. Introduction. Typically, if a foliation has one noncompact leaf, it has many noncompact leaves. In [1], Hurder inquired about how small the set of noncompact leaves in a foliation could be, and asked what features a foliation with few compact leaves would have. A foliation is called compact if all leaves are compact, and essentially compact if the set of noncompact leaves has zero Lebesgue measure. Here we consider the qualitative behaviour of essentially compact foliations that are not compact.

An example of an essentially compact foliation is in Reeb's thesis [4]. Reeb included the example to show that the global Reeb stability theorem for codimension one foliations could not be extended to higher codimensions. In Reeb's example, every leaf is proper and the closure of the set of noncompact leaves forms a compact submanifold. Vogt has generalized Reeb's construction in [3].

We offer some new examples, showing the following

THEOREM 1.1. There exists a $C^{\infty}$ essentially compact foliation of a compact manifold so that the closure of the set of noncompact leaves is not a submanifold. Also, there exists a $C^{\infty}$ essentially compact foliation of a compact manifold so that there are nonproper noncompact leaves.

We give the first example in Section 3 and the second example in Section 4.

In all of these examples, the set of noncompact leaves is uncountable. Vogt has shown that this must be the case for codimension two foliations with at least one noncompact leaf, and for oriented foliations of arbitrary codimension satisfying an additional cohomological condition [3].

It would be interesting to find a single general construction that yielded many examples of essentially compact foliations as well as that gave one or more of the known examples as special cases.

2000 Mathematics Subject Classification. 57R30.

Key words and phrases. Noncompact leaves, essentially compact foliation. 
We are grateful to Larry Conlon for his interest and helpful suggestions.

2. Reeb's example. Let $\mathcal{M}_{n}$ be the product $S^{n} \times S^{1} \times S^{1}$. We will view $S^{n}$ as the submanifold $\sum_{i=1}^{n+1} x_{i}^{2}=1$ of $\boldsymbol{R}^{n+1}$. For a point $p$ in $\mathcal{M}_{n}$, let $x_{1}(p)$ denote the projection to the $x_{1}$ coordinate in $\boldsymbol{R}^{n+1}$. Let $\phi(p)$ and $\theta(p)$ denote the projections of $p$ to the first and the second $S^{1}$ factors, respectively.

Define the differential forms

$$
\begin{aligned}
& \omega_{1}=d \theta, \\
& \omega_{2}=\left[(1-\sin \theta)^{2}+x_{1}^{2}\right] d \phi+\sin \theta d x_{1} .
\end{aligned}
$$

It may be checked that the $\omega_{1}$ and $\omega_{2}$ are nondegenerate and everywhere independent. Now define a distribution on $\mathcal{M}_{n}$ by $\omega_{1}=0$ and $\omega_{2}=0$. This system is integrable and hence yields a foliation $\mathcal{F}_{n}$ of $\mathcal{M}_{n}$.

Note that the submanifolds $\mathcal{N}_{c}$ defined by $\theta=c$, where $c$ is some constant, are saturated by leaves of $\mathcal{F}_{n}$. As long as $\sin \theta$ is not equal to one, all leaves of $\mathcal{F}_{n}$ in $\mathcal{N}_{c}$ are compact. They are given by the equations

$$
\begin{aligned}
\phi-\phi_{0} & =\frac{-\sin \theta}{1-\sin \theta} \arctan \left(\frac{x_{1}}{1-\sin \theta}\right) \quad \text { when } \sin \theta \neq 0, \\
\phi & =\phi_{0} \quad \text { when } \quad \sin \theta=0 .
\end{aligned}
$$

When $\sin \theta$ is equal to one, all leaves of $\mathcal{F}_{n}$ in $\mathcal{N}_{\pi / 2}$ except two are noncompact. The leaves in $\mathcal{N}_{\pi / 2}$ are given by

$$
\begin{aligned}
\phi-\phi_{0} & =\frac{1}{x_{1}} \quad \text { when } \quad x_{1} \neq 0, \\
x_{1} & =0 .
\end{aligned}
$$

The noncompact leaves in $\mathcal{N}_{\pi / 2}$ are all proper and have the submanifold $\mathcal{N}_{\pi / 2}$ as their closure.

We can view this foliation as arising from the one-parameter family of foliations of the submanifolds $\mathcal{N}_{c}$, where the family is indexed by $\theta=c$ in $S^{1}$. Every foliation in the family is a compact foliation, except the special foliation of the leaf $\mathcal{N}_{\pi / 2}$.

3. Second example. In this section, we describe an example of a dimension one foliation of a compact three-dimensional manifold so that the set of noncompact leaves is nonempty with Lebesgue measure zero. In this example, the closure of the set of noncompact leaves does not form a submanifold. The foliation is not a one-parameter family of foliations as is Reeb's example, although it is obtained from a one-parameter family of foliations of $[-1,1] \times \boldsymbol{R}$ by identifying the boundary with itself and adding a foliated $[-1,1] \times S^{1}$ as a limit leaf to make the resulting manifold compact.

3.1. The basic idea. Let $\mathcal{M}$ be the manifold with boundary given by $[-1,1] \times S^{1} \times$ $[-1,1]$. We will construct a one-dimensional foliation of $\mathcal{M}$ and then identify the boundary of $\mathcal{M}$ with itself to get a foliation $\mathcal{F}$ of a compact three-dimensional manifold without 
boundary. Let $(r, \theta, z)$ denote the coordinates on $\mathcal{M}$. We regard $\mathcal{M}$ as a thickening of the two-dimensional cylinder

$$
\mathcal{R}_{0}=\left\{(0, \theta, z) \mid \theta \in S^{1}, z \in[-1,1]\right\} .
$$

We will eventually identify the top

$$
\mathcal{T}=\left\{(r, \theta, 1) \mid r \in[-1,1], \theta \in S^{1}\right\}
$$

with the bottom

$$
\mathcal{B}=\left\{(r, \theta,-1) \mid r \in[-1,1], \theta \in S^{1}\right\} .
$$

Also, the inner side

$$
\mathcal{R}_{-1}=\left\{(-1, \theta, z) \mid \theta \in S^{1}, z \in[-1,1]\right\}
$$

will be identified with the outer side

$$
\mathcal{R}_{1}=\left\{(1, \theta, z) \mid \theta \in S^{1}, z \in[-1,1]\right\} .
$$

Let $\rho: \boldsymbol{R} \rightarrow S^{1}$ be the map taking $x$ in $\boldsymbol{R}$ to $x \bmod 2 \pi$ in $S^{1}$. We first take

$$
L_{1}^{0}=\{(0, \rho(\phi), \tanh (\phi+\pi)) \mid \phi \in \boldsymbol{R}\}
$$

as a single proper noncompact leaf for $\mathcal{F}$. Its closure contains the union of the circles

$$
Z_{1}=\{(0, t, 1) \mid t \in \boldsymbol{R}\}=\mathcal{T} \cap \mathcal{R}_{0}
$$

and

$$
Z_{-1}=\{(0, t,-1) \mid t \in \boldsymbol{R}\}=\mathcal{B} \cap \mathcal{R}_{0},
$$

so we add these as leaves as well.

For each $\varepsilon$ in $(-1,1]$, define a submanifold $\mathcal{S}_{\varepsilon}$ by

$$
\mathcal{S}_{\varepsilon}=\{(r, \rho(\phi), \tanh (\phi+\varepsilon \pi)) \mid r \in[-1,1], \phi \in \boldsymbol{R}\} .
$$

Each $\mathcal{S}_{\varepsilon}$ is a long spiraling strip homeomorphic in the submanifold topology to $\boldsymbol{R} \times[-1,1]$. The manifold $\mathcal{M}$ coincides with the union of the top $\mathcal{T}$, the bottom $\mathcal{B}$ and all of the submanifolds $\mathcal{S}_{\varepsilon}$.

The noncompact leaf $L_{1}^{0}$ is contained in the submanifold $\mathcal{S}_{1}$. This submanifold will be saturated by leaves of the foliation. For all $\varepsilon$ in $(-1,1)$, the submanifold $\mathcal{S}_{\varepsilon}$ is saturated by leaves of the foliation. As $|\varepsilon|$ gets close to one, the angle at which the leaves pierce the cylinder $\mathcal{R}_{0}$ should approach zero. Both the top $\mathcal{T}$ and bottom $\mathcal{B}$ will also be saturated sets for the foliation.

Lastly, we identify the boundary of $\mathcal{M}$ with itself in such a way that the foliation becomes a foliation of the resulting space. The resulting manifold will be a compact, nonorientable three-dimensional manifold that is doubly covered by the three torus.

3.2. Explicit description. In this section we elaborate and formalize the description just given.

Let

$$
\mathcal{M}^{\prime}=\mathcal{M} \backslash\left(\mathcal{B} \cup \mathcal{T} \cup \mathcal{S}_{1}\right)
$$


be the complement of $\mathcal{M}$ and the union of the top, the bottom, and the two-dimensional spiraling submanifold which contains the proper leaf $L_{1}^{0}$.

After removing $\mathcal{B} \cup \mathcal{T} \cup \mathcal{S}_{1}$ from $\mathcal{M}$, a topological cube remains. We parametrize it with the coordinates $(r, \phi, \varepsilon)$ as

$$
\mathcal{M}^{\prime}=\{(r, \phi, \tanh (\phi+\varepsilon \pi)) \mid r \in[-1,1], \varepsilon \in(-1,1), \phi \in \boldsymbol{R}\} .
$$

The use of $r, \phi$ and $\varepsilon$ is consistent with the previous use. Note that

$$
\frac{\partial}{\partial \phi}=\frac{\partial}{\partial \theta}+\left(1-z^{2}\right) \frac{\partial}{\partial z},
$$

so that the vector field $\partial / \partial \phi$ may be extended smoothly to all of $\mathcal{M}$. Note also that

$$
\frac{\partial}{\partial \varepsilon}=\pi\left(1-z^{2}\right) \frac{\partial}{\partial z} \text {. }
$$

First we define a vector field $\boldsymbol{F}_{\mathbf{0}}$ on $\mathcal{M}$. Let $f$ be a $C^{\infty}$ function defined on the real numbers $\boldsymbol{R}$ such that (i) $f$ is even, (ii) $f(0)=0$, (iii) $f$ increases from $r=0$ to $r=1 / 2$, (iv) $f(1 / 2)=1$, (v) $f$ decreases from $r=1 / 2$ to $r=1$, and (vi) $f \equiv 0$ for all $r$ with $|r| \geq 1$. We define $\boldsymbol{F}_{\mathbf{0}}$ on $\mathcal{M}$ by

$$
\boldsymbol{F}_{\mathbf{0}}(r, \theta, z)=f(r) \frac{\partial}{\partial r}+\frac{\partial}{\partial \phi} .
$$

The vector field $\boldsymbol{F}_{\mathbf{0}}$ is tangential to the top $\mathcal{T}$, the bottom $\mathcal{B}$, the cylinder $\mathcal{R}_{0}$ and each submanifold $\mathcal{S}_{\varepsilon}$. Let $\mathcal{F}_{0}$ denote the foliation of $\mathcal{M}$ integral to the vector field $\boldsymbol{F}_{\mathbf{0}}$.

The foliation $\mathcal{F}_{0}$ is tangential to the sides $\mathcal{R}_{-1}$ and $\mathcal{R}_{1}$, and we may turbulize it within distance $1 / 4$ from the sides to obtain a foliation $\mathcal{F}_{1}$ which is perpendicular to the boundary $\partial \mathcal{M}$ along the sides and tangential to $\partial \mathcal{M}$ at the top and bottom. Let $p$ be a $C^{\infty}$ bump function such that (i) $p$ is even, (ii) $p$ is identically zero for $|r| \leq 3 / 4$, (iii) $p$ increases for $3 / 4<r<1$, and (iv) $p$ is identically one for $|r| \geq 1$. Define a new vector field $\boldsymbol{F}_{\mathbf{1}}$ by

$$
\boldsymbol{F}_{\mathbf{1}}(r, \phi, \varepsilon)=(1-p(r)) \boldsymbol{F}_{\mathbf{0}}+p(r)\left[\cos \left(\frac{p(r) \pi}{2}\right) \frac{\partial}{\partial \phi}+\sin \left(\frac{p(r) \pi}{2}\right) \frac{\partial}{\partial r}\right] .
$$

Note that $\boldsymbol{F}_{\mathbf{1}}$ equals $\boldsymbol{F}_{\mathbf{0}}$ when $|r| \leq 3 / 4$ and is $C^{\infty}$ tangent to $\partial / \partial r$ at the sides $\mathcal{R}_{-1}$ and $\mathcal{R}_{1}$ of $\mathcal{M}$. The vectors $\partial / \partial \phi$ and $\partial / \partial r$ are tangential to the submanifolds $\mathcal{S}_{\varepsilon}$, so that the foliation $\mathcal{F}_{1}$ integral to $\boldsymbol{F}_{\mathbf{1}}$ is also tangential to $\mathcal{S}_{\varepsilon}$ for all $\varepsilon$.

We now define a $C^{\infty}$ bump function $h$ to use in defining a perturbation $\boldsymbol{F}$ of $\boldsymbol{F}_{\mathbf{1}}$. First we must define some other $C^{\infty}$ bump functions. Let $s$ be a $C^{\infty}$ bump function defined on $\boldsymbol{R}$ such that (i) $s$ is even, (ii) $s$ is identically zero for $x$ with $|x| \geq 1$, (iii) $s$ is increasing from $x=-1$ to $x=0$, and (iv) $s(0)=1 / 2$. Let

$$
G=\{(r, \varepsilon)|| r \mid \leq s(\varepsilon)\} .
$$

Define a $C^{\infty}$ function $g$ with domain $[-1,1] \times[-1,1]$ whose support is $G$ such that (i) $g$ is even with respect to $r$ and $\varepsilon$, (ii) $g(0,0)=1$, and (iii) $g$ decreases on radial paths from $(0,0)$ 
to the boundary of $G$. We define $h$ by

$$
h(r, \phi, \varepsilon)=\left\{\begin{array}{lll}
e^{-\phi^{2}} g\left(r \phi^{2}, \varepsilon\right) & \text { on } & \mathcal{M}^{\prime}, \\
0 & \text { on } & \mathcal{M} \backslash \mathcal{M}^{\prime} .
\end{array}\right.
$$

In the next section, we will show that $h$ is smooth on $\mathcal{M}$. The support $H$ of $h$ is the closure in $\mathcal{M}$ of the set

$$
\left\{(r, \phi, \varepsilon)|| r \mid \leq s(\varepsilon) / \phi^{2}\right\} .
$$

The interior of this set is an open neighborhood of $\mathcal{R}_{0} \backslash L_{1}^{0}$ in $\mathcal{M} \backslash \mathcal{M}^{\prime}$. As $|z|$ goes to one, the $r$ coordinates of points in $H$ approach zero. The intersection of $H$ with $\mathcal{M} \backslash \mathcal{M}^{\prime}$ is equal to the union of $L_{0}^{1}, Z_{1}$ and $Z_{-1}$.

We define a new vector field $\boldsymbol{F}$ as a perturbation of $\boldsymbol{F}_{\mathbf{1}}$ by

$$
\boldsymbol{F}(r, \phi, \varepsilon)=\boldsymbol{F}_{\mathbf{1}}+h(r, \phi, \varepsilon) \frac{\partial}{\partial r} .
$$

Let $\mathcal{F}$ be the foliation integral to $\boldsymbol{F}$. The vector field $\boldsymbol{F}$ is tangential to $\mathcal{S}_{\varepsilon}$ for all $\varepsilon$, so that $\mathcal{S}_{\varepsilon}$ is saturated by leaves of $\mathcal{F}$ for all $\varepsilon$.

Identify the boundary of $\mathcal{M}$ with itself according to the equivalence relation

$$
(r, \theta, z) \sim(-r,-\theta,-z) .
$$

Note that the boundary of the submanifold $\mathcal{S}_{\varepsilon}$ is identified with the boundary of $\mathcal{S}_{-\varepsilon}$, since

$$
(1, \phi, \tanh (\phi+\varepsilon \pi)) \sim(-1,-\phi, \tanh (-\phi-\varepsilon \pi)),
$$

and the top $\mathcal{T}$ and bottom $\mathcal{B}$ are identified according to the relation

$$
(r, \theta, 1) \sim(-r,-\theta,-1) .
$$

The vector field $\boldsymbol{F}$ has appropriate symmetry so that the distribution it spans is mapped to itself by the identifications.

3.3. Properties of the example. In this section, we demonstrate that the foliation $\mathcal{F}$ is an example of the first type in Theorem 1.1. First we show the symmetry of the foliation.

LEMma 3.1. Suppose that the identifications of the boundary have not yet been made. Suppose the leaf $L_{\varepsilon}$ of $\mathcal{F}$ is contained in $\mathcal{S}_{\varepsilon}$. Then it has boundary points

$$
(-1, a, \tanh (a+\varepsilon \pi)) \text { and }(1, b, \tanh (b+\varepsilon \pi)),
$$

for some real numbers $a$ and $b$. In addition, there is another leaf $L_{-\varepsilon}^{-}$of $\mathcal{F}$ contained in $\mathcal{S}_{-\varepsilon}$ which has boundary points

$$
(-1,-b, \tanh (-b-\varepsilon \pi)) \text { and }(1,-a, \tanh (-a-\varepsilon \pi)) .
$$

Proof. The vector field $\boldsymbol{F}$ has the property that

$$
\boldsymbol{F}(r, \phi, \varepsilon)=\boldsymbol{F}(-r,-\phi,-\varepsilon) .
$$


Consider the restriction of $\boldsymbol{F}$ to $\mathcal{S}_{\varepsilon}$. The coefficient of $\partial / \partial \phi$ in $\boldsymbol{F}$ is always positive when $|r|<1$, and hence any leaf $L_{\varepsilon}$ in $\mathcal{S}_{\varepsilon}$ may be written as a graph

$$
L_{\varepsilon}=\{(r(\phi), \phi, \tanh (\phi+\varepsilon \pi)) \mid \phi \in[a, b]\} .
$$

The graph is over a compact interval because the coefficient of $\partial / \partial r$ in $\boldsymbol{F}$ is positive for all points in $\mathcal{M}^{\prime}$.

By the symmetry of $\boldsymbol{F}$, there is another leaf $L_{-\varepsilon}^{-}$contained in $\mathcal{S}_{-\varepsilon}$ which may be written as

$$
L_{-\varepsilon}^{-}=\{(-r(\phi),-\phi, \tanh (-\phi-\varepsilon \pi)) \mid \phi \in[a, b]\} .
$$

The boundary points of $L_{\varepsilon}$ are

$$
(-1, a, \tanh (a+\varepsilon \pi)) \text { and }(1, b, \tanh (b+\varepsilon \pi)),
$$

and the boundary points of $L_{-\varepsilon}^{-}$are

$$
(-1,-b, \tanh (-b-\varepsilon \pi)) \text { and }(1,-a, \tanh (-a-\varepsilon \pi)),
$$

proving the lemma.

Next we show that the foliation is essentially compact.

LEMMA 3.2. The union of noncompact leaves of $\mathcal{F}$ forms a set of Lebesgue measure zero.

Proof. The saturated open set $\mathcal{M}^{\prime}$ has full measure. We will show that all of the leaves of $\mathcal{F}$ contained in $\mathcal{M}^{\prime}$ are compact. For any submanifold $\mathcal{S}_{\varepsilon}$, where $\varepsilon \in(-1,1)$, by Lemma 3.1, every leaf of $\mathcal{F}$ in $\mathcal{S}_{\varepsilon}$ is homeomorphic to a closed interval, with one boundary component with $r=-1$ and one boundary component with $r=1$.

After boundary points are identified, the boundary points of the leaf $L_{\varepsilon}$ of $\mathcal{S}_{\varepsilon}$ are identified with those of the leaf $L_{-\varepsilon}^{-}$of $\mathcal{S}_{-\varepsilon}$ so that the union of the two leaves forms a compact leaf of the final foliation.

Suppose the leaves $L_{\varepsilon}$ and $L_{-\varepsilon}^{-}$are as in Lemma 3.1. Consider the leaf $L_{\varepsilon}$ after identifications of the boundary are made. The boundary point $(1, b, \tanh (b+\varepsilon \pi))$ is identified with the point

$$
(-1,-b,-\tanh (b+\varepsilon \pi))=(-1,-b, \tanh (-b-\varepsilon \pi)),
$$

a boundary point of the leaf $L_{-\varepsilon}^{-}$contained in $\mathcal{S}_{-\varepsilon}$. The other boundary point $(1,-a$, $\tanh (-a-\varepsilon \pi))$ of $L_{-\varepsilon}^{-}$is identified with

$$
(-1, a,-\tanh (-a-\varepsilon \pi))=(-1, a, \tanh (a+\varepsilon \pi)),
$$

which is the second boundary component of the original leaf $L_{\varepsilon}$.

In the case that the leaf $L$ goes through the point $(0,0,0)$, the two boundary points of $L$ are identified, and hence $L$ alone becomes a compact leaf.

The next lemma describes the set of noncompact leaves. 
LEMMA 3.3. The closure of the set of noncompact leaves is nonempty, has Lebesgue measure zero and is not a submanifold. There is an uncountable number of noncompact leaves.

Proof. The closure of the set of noncompact leaves is $\mathcal{M} \backslash \mathcal{M}^{\prime}$, which is clearly not an embedded submanifold. The foliation $\mathcal{F}$ agrees with the foliation $\mathcal{F}_{1}$ on the set $\mathcal{M} \backslash \mathcal{M}^{\prime}$, which is a saturated closed set for both foliations. All leaves of the foliation $\mathcal{F}_{1}$ contained in $\mathcal{M} \backslash \mathcal{M}^{\prime}$ are noncompact except for $L_{1}^{0}$, the circles $Z_{1}$ and $Z_{-1}$, and the four circular boundary components of $\mathcal{T}$ and $\mathcal{B}$.

Before demonstrating that the foliation is smooth, we compute partial derivatives of the function $h$.

LEMMA 3.4. Suppose that $m, n$ and $p$ are nonnegative integers. For a point $(r, \phi, \varepsilon)$ in $\mathcal{M}^{\prime}$, the quantity

$$
\frac{\partial^{m+n+p} h}{\partial z^{m} r^{n} \phi^{p}}(r, \phi, \varepsilon)
$$

is the sum of terms of the form

$$
C \frac{z^{g}}{\left(1-z^{2}\right)^{h}} e^{-\phi^{2}} \phi^{i} r^{j} \frac{\partial^{k+l} g}{\partial \varepsilon^{k} \partial r^{l}}\left(\phi^{2} r, \varepsilon\right),
$$

where $C$ is a constant and $g, h, i, j, k$ and $l$ are nonnegative integers.

PROOF. First we assume that $m=0$. From the definition of $h$, the result clearly holds if $n=p=0$. A short calculation shows that

$$
\begin{aligned}
& \frac{\partial h}{\partial r}(r, \phi, \varepsilon)=\phi^{2} e^{-\phi^{2}} \frac{\partial g}{\partial r}\left(\phi^{2} r, \varepsilon\right), \quad \text { and } \\
& \frac{\partial h}{\partial \phi}(r, \phi, \varepsilon)=2 \phi e^{-\phi^{2}}\left(-g\left(\phi^{2} r, \varepsilon\right)+r \frac{\partial g}{\partial r}\left(\phi^{2} r, \varepsilon\right)\right),
\end{aligned}
$$

demonstrating the claim if $m=0$ and $n+p=1$.

If we differentiate $h$ with respect to $\phi$ or $r$ again, we will only introduce polynomial $\phi$ terms, $r$ terms and higher order partial derivatives of $g$. Thus, the higher order derivatives of $h$ with respect to $r$ and $\phi$ are given by the sum of terms of the form

$$
C e^{-\phi^{2}} \phi^{i} r^{j} \frac{\partial^{k+l} g}{\partial \varepsilon^{k} \partial r^{l}}\left(\phi^{2} r, \varepsilon\right)
$$

where $C$ is a constant and $i, j, k$ and $l$ are nonnegative integers. Thus the lemma holds when $m=0$.

Now we assume that $m>0$. We obtain the derivative by first differentiating with respect to $r$ and $\phi$ to get a sum of terms of the form of expression (3.3). Using the fact that $\partial / \partial \varepsilon=$ $\pi\left(1-z^{2}\right) \partial / \partial z$, it is easily seen that the partial derivative of such a term with respect to $z$ is given by

$$
\frac{\partial}{\partial z}\left[C e^{-\phi^{2}} \phi^{i} r^{j} \frac{\partial^{k+l} g}{\partial \varepsilon^{k} \partial r^{l}}\left(\phi^{2} r, \varepsilon\right)\right]=\frac{1}{\pi\left(1-z^{2}\right)} C e^{-\phi^{2}} \phi^{i} r^{j} \frac{\partial^{k+l+1} g}{\partial \varepsilon^{k+1} \partial r^{n}}\left(\phi^{2} r, \varepsilon\right) .
$$


Thus the lemma holds when $m=1$.

If we differentiate with respect to $z$ again, using the product rule we get a sum of terms of the appropriate form. Consequently, the lemma holds for arbitrary $m$.

We will use the following lemma from Calculus to show that the function $h$ is smooth. The proof is elementary, and so we omit it.

LEMMA 3.5. Let $h$ and $i$ be nonnegative integers, and let $z=\tanh (\phi+\varepsilon \pi)$. Then

$$
\lim _{|z| \rightarrow 1} \frac{e^{-\phi^{2}} \phi^{i}}{\left(1-z^{2}\right)^{h}}=0 .
$$

The vector field $\boldsymbol{F}_{\mathbf{1}}$ is clearly $C^{\infty}$. To show that $\boldsymbol{F}$ is $C^{\infty}$, it suffices to show that the function $h$ is $C^{\infty}$.

LEMMA 3.6. The function $h$ is $C^{\infty}$.

PROOF. Let $\gamma$ be a path in the support $H$ of $h$ such that the points $\gamma(t)$ are in $\mathcal{M}^{\prime}$ for $t$ in $\left[0, t_{0}\right)$, and the point $\gamma\left(t_{0}\right)$ is in $\mathcal{M} \backslash \mathcal{M}^{\prime}$. We will show that for all nonnegative $m, n$ and $p$,

$$
\left.\lim _{t \rightarrow t_{0}} \frac{\partial^{m+n+p} h}{\partial z^{m} \partial r^{n} \partial \phi^{p}}\right|_{\gamma(t)}=0 .
$$

The function $h$ is defined to be equal to zero on $\mathcal{M} \backslash \mathcal{M}^{\prime}$, and hence this will prove the lemma.

Because the intersection of $H$ and $\mathcal{M} \backslash \mathcal{M}^{\prime}$ equals $L_{1}^{0} \cup Z_{1} \cup Z_{-1}$, we are concerned with two cases: the case that $\gamma\left(t_{0}\right)$ is in $L_{1}^{0}$, and the case that $\gamma\left(t_{0}\right)$ is in $Z_{1} \cup Z_{-1}$.

Case 1. The limiting point $\gamma\left(t_{0}\right)$ is in $L_{1}^{0}$.

The $z$ coordinate of $\gamma\left(t_{0}\right)$ is between -1 and 1 , so that there is some number $c$ in $\left[0, t_{0}\right)$ such that for all points $\gamma(t)$ with $t$ in $\left[c, t_{0}\right)$, the quantity

$$
C \frac{z^{g}}{\left(1-z^{2}\right)^{h}} e^{-\phi^{2}} \phi^{i} r^{j}
$$

is bounded. For $m, n \geq 0$, the quantity

$$
\left.\frac{\partial^{k+l} g}{\partial \varepsilon^{k} \partial r^{l}}\left(r \phi^{2}, \varepsilon\right)\right|_{\gamma(t)}
$$

approaches zero as $t$ goes to $t_{0}$ and $\left(r \phi^{2}, \varepsilon\right)$ approaches the boundary of $G$. Thus, a term of the form (3.2) goes to zero, and hence all partial derivatives of $h$ go to zero as $t$ goes to $t_{0}$.

Case 2. The limiting point $\gamma\left(t_{0}\right)$ is in $Z_{1} \cup Z_{-1}$.

The quantity

is bounded over $\mathcal{M}$.

$$
C z^{g} r^{j} \frac{\partial^{k+l} g}{\partial \varepsilon^{k} \partial r^{l}}
$$

As $t$ approaches $t_{0}$, the absolute value of the $z$-coordinate of $\gamma(t)$ approaches 1 . Applying Lemma 3.5, we see that for $\gamma(t)$, a term of the form shown in expression (3.2) goes to zero as $t$ approaches $t_{0}$. Hence all partial derivatives of $h$ go to zero as $t$ approaches $t_{0}$. 
We have shown that $\mathcal{F}$ is a $C^{\infty}$ foliation such that the set of noncompact leaves is nonempty, not a submanifold, and of Lebesgue measure zero.

We may unite this example with Reeb's by viewing them in the following way. The manifold $\mathcal{M}_{n}$ in Reeb's example is foliated by the manifolds $\mathcal{N}_{\theta}$. This is a proper foliation, and each leaf $\mathcal{N}_{\theta}$ is saturated by leaves of the original foliation. For all leaves $\mathcal{N}_{\theta}$ except one, the restriction of the original foliation of $\mathcal{N}_{\theta}$ is a compact foliation. In the second example, we get a two-dimensional foliation $\mathcal{F}^{\varepsilon}$ of $\mathcal{M}$ from the submanifolds $\mathcal{S}_{\varepsilon}$ and $\mathcal{B}$ after boundary identifications. The foliation $\mathcal{F}^{\varepsilon}$ of $\mathcal{M}$ is a proper foliation, with each leaf for $\mathcal{F}^{\varepsilon}$ saturated by leaves of the original foliation $\mathcal{F}$. For all leaves $L_{\varepsilon}$ of $\mathcal{F}^{\varepsilon}$ except those coming from $\mathcal{B}$ and $\mathcal{S}^{1}$, the restriction of the foliation $\mathcal{F}$ to $L_{\varepsilon}$ is a compact foliation. So, to generalize the construction of the second example, we would want to find a proper foliation that is saturated by leaves of a foliation of higher codimension so that the restricted foliations are almost always compact foliations.

4. A variation of an example of Sullivan. In the two examples described so far, all leaves of the foliations are proper. By making a small alteration to Sullivan's counterexample to the periodic orbit conjecture [2], we construct an example of a foliation with nonproper leaves such that the set of noncompact leaves has measure zero, demonstrating the second part of Theorem 1.1.

Let $G$ be the group of matrices of the form

$$
\left(\begin{array}{lll}
1 & x & y \\
0 & 1 & z \\
0 & 0 & 1
\end{array}\right),
$$

where $x, y$ and $z$ are real numbers. Let $\Gamma$ be the subgroup of $G$ consisting of elements with integer entries. The left-invariant forms on $G$ are spanned by the elements $d x, d y$ and $\eta=d z-x d y$.

Let $\mathcal{M}$ equal the product $G / \Gamma \times S^{1} \times S^{1}$. Let $\alpha$ and $\beta$ be the coordinates in the first and the second $S^{1}$ factors, respectively. Define the vector field $Y$ on $G / \Gamma$ by

$$
\begin{aligned}
d x(Y) & =(\sin (2 \beta)) \cos \alpha, \\
d y(Y) & =-(\sin (2 \beta)) \sin \alpha+2 \cos ^{2} \beta \cos \alpha, \\
\eta(Y) & =\cos ^{2} \beta, \quad d \alpha(Y)=2 \sin ^{2} \beta, \quad d \beta(Y)=0 .
\end{aligned}
$$

Note that integral curves saturate the submanifolds $\beta=c$, where $c$ is constant, so as in Reeb's example, we will get a one-parameter family of foliations indexed by $\beta$.

Then, when $\beta \neq 0$ and $\beta \neq \pi$, integral curves of $Y$ satisfy

$$
\begin{aligned}
& \alpha=\lambda t+\alpha_{0}, \\
& x=(\cot \beta)\left[\sin \left(\lambda t+\alpha_{0}\right)-\sin \alpha_{0}\right]+x_{0}, \\
& y=(\cot \beta)\left[\cos \left(\lambda t+\alpha_{0}\right)-\cos \alpha_{0}\right]+\cot ^{2} \beta \sin \left(\lambda t+\alpha_{0}\right)+y_{0}, \\
& \int_{0}^{2 \pi / \lambda} d z(Y)=\int_{0}^{2 \pi / \lambda} \eta(Y)+\int_{0}^{2 \pi / \lambda} x d y(Y)=0,
\end{aligned}
$$


where $\lambda=2 \sin ^{2} \beta$. So, when $\beta$ is not equal to 0 or $\pi$, all leaves are compact.

When $\beta$ equals 0 or $\pi$, we obtain

$$
d x(Y)=0, \quad d y(Y)=2 \cos \alpha, \quad \eta(Y)=1, \quad d \alpha(Y)=0, \quad d \beta(Y)=0 .
$$

Solutions are given by

$$
\begin{gathered}
x=x_{0}, \quad y=\left(2 \cos \alpha_{0}\right) t+y_{0}, \quad z=\left(1+2 x_{0} \cos \alpha_{0}\right) t+z_{0}, \\
\alpha=\alpha_{0}, \quad \beta=\beta_{0} .
\end{gathered}
$$

When the ratio of $2 \cos \alpha_{0}$ to $1+2 x_{0} \cos \alpha_{0}$ is irrational, the leaf is noncompact and dense in the submanifold given by

$$
x=x_{0}, \quad \alpha=\alpha_{0}, \quad \beta=\beta_{0},
$$

and hence is nonproper. When the ratio is rational, all leaves are compact.

\section{REFERENCES}

[1] R. LAngevin, A list of questions about foliations, Differential topology, foliations, and group actions (Rio de Janeiro, 1992), 59-80, Contemp. Math. 16, Amer. Math. Soc., Providence, RI, 1994.

[2] D. Sullivan, A counterexample to the periodic orbit conjecture, Inst. Hautes Études Sci. Publ. Math. 46 (1976), 5-14.

[ 3 ] E. Vogt, Foliations with few non-compact leaves, Algebr. Geom. Topol. 2 (2002), 257-284.

[ 4 ] W. T. Wu And G. REeb, Sur les éspaces fibres et les variétés feuillitées, Hermann, 1952.

DEPARTMENT OF MATHEMATICS

IDAHO STATE UNIVERSITY

POCATELLO, ID 83209

U.S.A.

E-mail address: tpayne@member.ams.org 